\title{
The influence of spatial irregularity upon radial-maze performance in the rat
}

\author{
M.-C. GROBÉTY and F. SCHENK \\ Institut de Physiologie Humaine, Lausanne, Switzerland
}

\begin{abstract}
In two experiments, we examined how the introduction of vertical or horizontal irregularities in the perfectly regular shape of a radial maze affected rats' performances. The introduction of various tilts in each arm of an eight-arm radial maze had a slightly positive effect on accuracy. However, when intra- and extramaze cues were dissociated by rotating the maze before maze completion, the rats relied preferentially on extramaze cues associated with the horizontal direction of the arms but not with the tilts. On the other hand, the rats showed poor performances when trained on a horizontally distorted maze (uneven angles between the arms instead of repeated $45^{\circ}$ angles). The high number of errors was related to the neglect of particular arms, the disorganization of the patrolling sequences, and the tendency to chain the visit of five arms that formed a regular shape. Other animals, trained in the same maze, displayed similar biases even after a pretraining phase with constrained choices. Results from the horizontally distorted maze confirm and extend data from the spontaneous alternation literature that choice behavior is influenced by rules of movement that favor large angle transitions and regular subdivisions of space. They also stress the relation between performance in the radial maze and spontaneous exploratory and foraging behaviors.
\end{abstract}

Since its introduction by Olton and Samuelson (1976), the radial maze has become a very popular test for the study of spatial memory in animals. The maze consists of a central arena from which arms radiate in a regular pattern similar to the spokes of a wheel. Rats are allowed to collect food located at the ends of the arms. The animals organize their foraging behavior efficiently, avoiding reentries to previously visited arms. Very high choice accuracy has been obtained in mazes with up to 17 arms (Olton, Collison, \& Werz, 1977) or even 24 arms in a hierarchical maze (Roberts, 1979). Furthermore, rats maintain their performance in spite of prolonged interruption in the middle of a test (Beatty \& Shavalia, 1980), following sensory perturbation during an interruption (Maki, Brokofsky, \& Berg, 1979), or even after trials in other radial mazes during the interruption (Cook \& Brown, 1985). Kraemer, Gilbert, and Innis (1983) have shown that rats rely preferentially on extramaze cues but are also capable of highly accurate choices when trained with intramaze cues, even when the intramaze configuration is altered on each trial. Moreover, blind rats are also able to maintain good performance in a radial maze when tested with an uninterrupted free-choice procedure (Zoladek \& Roberts, 1978).

According to these results, choice accuracy in the radial maze appears remarkably resistant to many treatments or

We are grateful to Marianne Gafner for her skillful help with training the rats and to $\mathbf{N}$. Berchtold for reviewing the English of our manuscript. Funding was provided by Grant 3.392-0.86 from the Swiss National Foundation for Scientific Research. Requests for reprints should be directed to F. Schenk, Institute of Physiology, School of Medicine, University of Lausanne, CH-1005 Lausanne, Switzerland. procedures. However, Brown (1990) recently demonstrated that a simple change in radial-maze arm length dramatically reduced the efficiency of the animals. This author indicated that the observed performances were probably not caused by poorer discrimination of the short arms but more likely resulted from a modification of the choice criterion for visiting short arms that either lowered fear or produced a difference in the cost/ benefit analysis of choices between short and long arms. In any case, this manipulation decreases the tendency to avoid revisits to the short arms. We recently observed that rats also performed poorly in a radial maze in which two adjacent arms were parallel (Schenk, Contant, \& Grobéty, 1990). Therefore, performance in the radial maze appears to be sensitive to the shape of the maze.

In the present series of experiments, we decided to test whether the regularity of the shape of the radial maze had an influence on the performance of the animals. Rats were tested in a standard radial maze and in two kinds of irregular mazes. Irregularity was introduced in the radial maze either by varying the horizontal angles separating the arms (horizontal component of irregularity) or by tilting the arms at different angles (vertical component of irregularity).

\section{EXPERIMENT 1}

\section{Method}

\section{Subjects}

The subjects were 24 male hooded Long-Evans rats, which were 3 to 5 months old at the beginning of the experiment. They were born in our breeding colony, where they were kept in groups of 4 , in glass cages $(40 \times 40 \times 50 \mathrm{~cm})$ containing large wooden nest boxes. They had been trained in a place learning task in the Morris 
water maze during development (3-5 weeks of age). Throughout all of the experiments, the rats were given free access to water, but they were kept on a diet designed to maintain their body weights at about $90 \%$ of free-feeding values. The daily food ration was given after the testing period.

\section{Apparatus}

The three radial mazes used in this experiment were built with the same elements. Eight Plexiglas tunnels $(12 \times 12 \times 60 \mathrm{~cm})$ were arranged on wooden supports $(12 \times 60 \mathrm{~cm})$ and positioned on a large rotating board to form a transparent enclosed radial maze. The central choice area (approximately $32 \mathrm{~cm}$ in diameter) was covered by a Plexiglas lid. Before each test, a drop of diluted condensed milk was placed on a small glass plate at the end of each arm. The arms were arranged in different positions to form the three different maze shapes. The first maze was a regular radial maze, with all arms horizontal and separated by $45^{\circ}$ angles (standard maze, Figure la). In the second maze, all of the arms were also horizontal, but the adjacent arms were separated by different angles, as shown in Figure lb (variable angle: VA maze). In the third maze, the arms remained separated by a horizontal $45^{\circ}$ angle, but the arms were tilted at different angles (variable tilt: VT maze, Figure 1c).

The experiments were conducted in a $4 \times 5 \mathrm{~m}$ room containing a heterogeneous collection of extramaze stimuli (shelves, sink, table and chairs, blackboard, pictures, etc.). The maze was indirectly lit by three $40-\mathrm{W}$ light bulbs directed toward the ceiling. The position of the board supporting the maze was constant in the testing room, but four orientations of the maze were used, the arm designated as number 1 pointing North, South, East, or West. Throughout the trials, the orientation of the maze remained constant for a given rat (i.e., arm number 1 was always pointing in the same direction).

\section{Procedure}

The rats were randomly assigned to one of three groups, each group being tested on either the standard maze, the VA maze, or the VT maze. No pretraining tests were conducted. The 24 anjmals were tested individually in random order, with one session per day.

Free-choice procedure. The three groups were first tested with a free-choice procedure for 12 days. An animal was placed in the central arena and allowed to choose arms until all eight arms had been visited. Three extra choices or $1 \mathrm{~min}$ of activity were given to the rats to avoid interrupting the exploration of the maze right after the last correct choice. The rat was then removed from the maze and placed in a holding bucket until the other animals had been tested.

"4-8 choice" procedure. Following the completion of the freechoice testing phase, the rats were tested by means of a modified free-choice procedure. Prior to each trial, the entrances of four arms, arbitrarily chosen for each test and each animal, were blocked by Plexiglas sliding doors. The tested rat was placed in the maze and allowed to make choices until the four unblocked arms had been visited. It was then removed from the maze and placed in a holding bucket for $1 \mathrm{~min}$. Meanwhile, the experimenter reopened the four blocked arms. The rat was then reintroduced to the center of the maze and allowed to choose among all eight arms until the four unvisited arms had been explored. Then, as in the free-choice procedure, three more choices were allowed (or $1 \mathrm{~min}$ of activity) before the animal was removed from the maze. The groups on the standard and the VT mazes were tested for 12 days with this procedure. Because of the slow learning rate observed in the VA maze, training without interruption was extended to 18 days for this group.

"4-8 choice" procedure with rotation. Finally, the groups run on the standard maze and on the VT maze were further tested for 6 days with a special $4-8$ choice procedure. During the interruption, a dissociation between the intra- and extramaze cues was induced by rotating the maze by either $\pm 45^{\circ}, \pm 90^{\circ}$, or $180^{\circ}$. After the interruption, the animals therefore had to complete the maze according to either the intramaze cues (odors and tilts for the irregular tilted maze) or to the extramaze ones. During the interruption, the experimenter baited the four remaining arms according to the intramaze cues for the first three trials and according to the extramaze cues for the next three trials.

Data analysis. The performance of the animals is described with regard to the number of errors (entries into already visited arms) made before obtaining the food in all arms. The last correct arm to be visited in each test was also analyzed, thus indicating a frequency of neglect for each specific arm of each maze shape.

The sequential choice tendency was analyzed by assigning a transition turn direction to all arm-to-arm transitions. A transition was considered as positive when the turn direction was clockwise and as negative when the turn direction was counterclockwise. When the transition angle was $180^{\circ}$, the turn direction was considered to be the same sign as the previous transition in the exploratory sequence. Within the first eight choices on a trial (seven turns), the number of times the animals changed their tum direction was also calculated.

\section{Results}

Free-choice procedure. The mean number of errors made prior to obtaining the food placed in each of the eight baited arms on the three mazes (standard, VA, and VT) is plotted as a function of three-trial blocks in Figure $2 \mathrm{a}$. All of the groups consistently improved their choice performance during the first phase of training. In

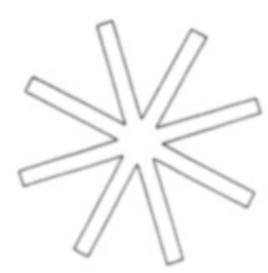

Standard Maze b

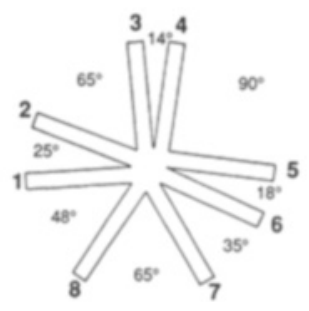

Irregular Maze

Irregular Maze
With Variable Horizontal Angles c

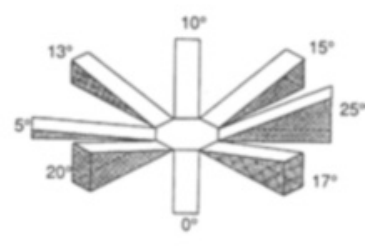

Irregular Maze

With Varriable Tilted Arms

Figure 1. Shapes of the three different radial mazes: (a) standard maze, (b) maze with variable angles (VA maze), and (c) maze with variable tilts (VT maze). 


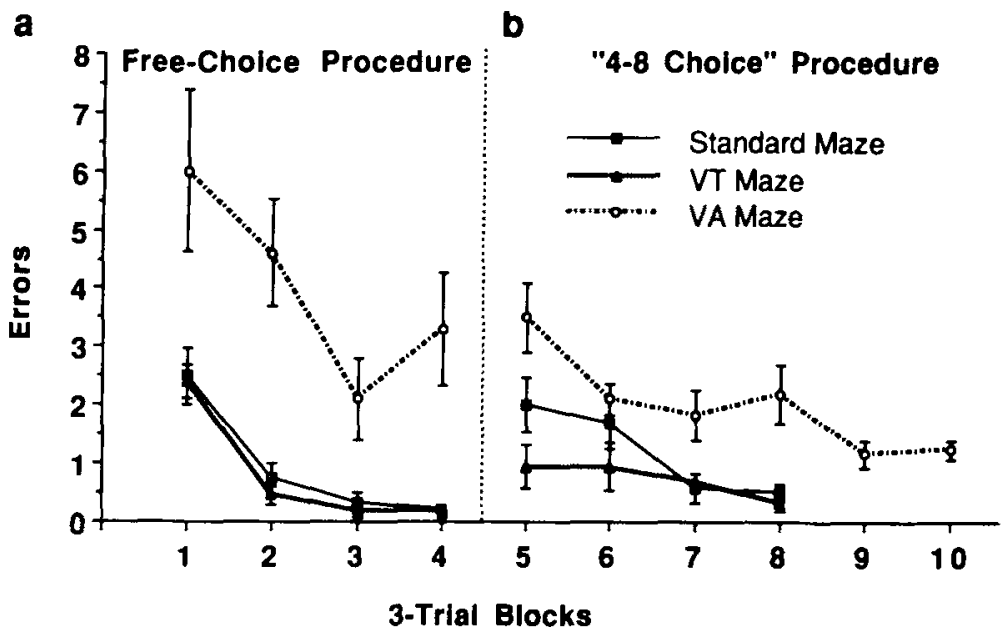

Figure 2. Mean number of errors in blocks of three trials on the different mazes during the free-choice procedure (Blocks 1-4) and the "4-8 choice" procedure (Blocks 5-10) of Experiment 1.

addition, there was a significant between-group difference in choice accuracy, with rats in the VA maze requiring more choices to complete the maze than did the animals in the two other groups [two-way analysis of variance (ANOVA); between groups, $F(2,20)=16.89, p<.001$; repetitions, $F(11,231)=5.45, p<.001]$.

Among the three groups, only the animals tested in the VA maze showed a nonrandom distribution of the last arm visited. Figure 3 shows that Arms 1, 3, or 6 were systematically visited last $\left\{\chi^{2}(7)=61.33, p<.001\right\}$. In addition, visits to the other five arms $(2,4,5,7$, and 8$)$ were frequently chained. Four or five of these arms appeared to be visited consecutively in the first eight choices in $49.6 \%$ of the tests. By contrast, the same sequences

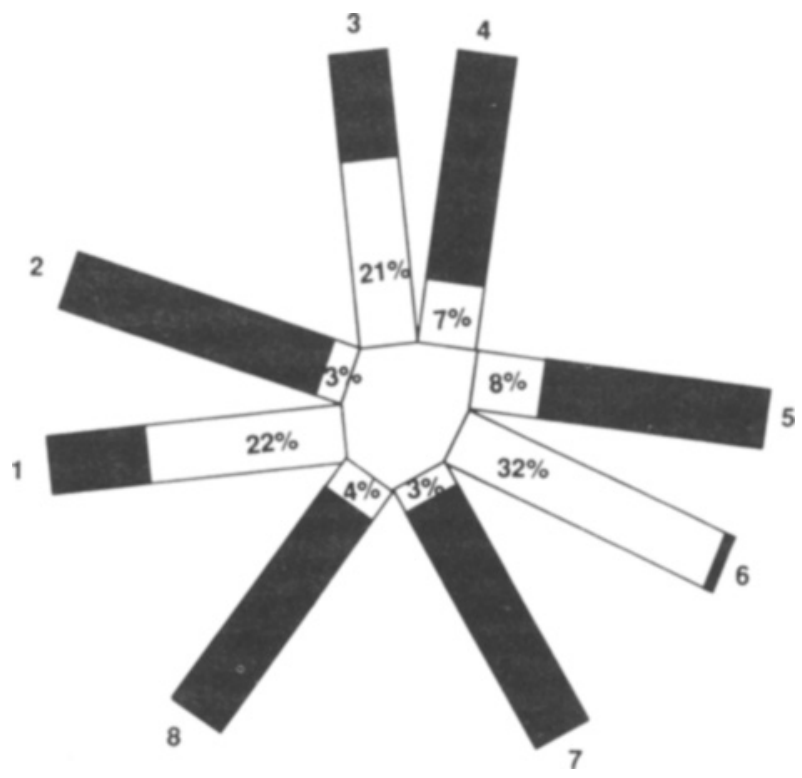

Figure 3. Distribution of the last arm found by the animals in the VA maze during the free-choice procedure of Experiment 1. of visits in the standard radial maze appeared in only $29.5 \%$ of the tests $\left[\chi^{2}(1)=22.72, p<.001\right]$.

The mean number of changes between clockwise and counterclockwise turn directions in the transitions of the first eight arms visited was $0.69 \pm 0.89$ on the standard maze, $0.60 \pm 0.14$ in the VT maze, and $1.24 \pm 0.15$ in the VA maze. A two-way ANOVA revealed that these changes were significantly more frequent in the VA maze than in the two others $[F(2,21)=4.05, p<.05]$ and that they decreased with training $[F(11,231)=4.57$, $p<.001]$.

"4-8 choice" procedure. Figure $2 b$ shows the mean number of errors observed during this second phase of training. The change of procedure increased the number of errors in all groups, but an ANOVA indicated that those errors were not significantly different for the standard and VT maze groups. However, the animals on the VT maze reached the criterion of no more than a single error in three consecutive trials in $3.9 \pm 1.4$ days, whereas the group on the standard maze reached this criterion significantly later, in $7.0 \pm 1.1$ trials [Mann-Whitney $U(8,7)=$ $13, p<.05]$.

Only half of the animals on the VA maze reached this criterion within the 18 days of training. Their mean number of errors remained relatively high, with about two errors per test. The majority of these errors occurred in the second part of the trials and consisted mainly of reentrances to arms visited before the interruption.

"4-8 choice" procedure with rotation. The mean number of errors in the standard maze was $5.04 \pm 0.68$ when reinforced on intramaze cues and $1.33 \pm 0.23$ when reinforced on extramaze ones. With the same procedures on the VT maze, the animals scored $4.48 \pm 0.54$ and $1.67 \pm 0.46$ errors, respectively. Both groups showed better performances when reinforced, after the rotation, on the extramaze cues than on the intramaze cues (Wilcoxon test; standard maze $Z=3.80, p<.001$; VT maze $Z=$ $3.45, p<.001$ ). When reinforced on the intramaze cues, 
the presence of the tilts as additional intramaze cues in the VT maze did not significantly facilitate performance of the animals (Mann-Whitney $Z=0.24, p=.81$ ) relative to the standard maze.

\section{Discussion}

There was clear evidence of an improvement in accuracy in all the groups throughout the free-choice procedure. Introducing an interruption after four choices increased the number of errors in all groups, but accuracy improved with further training. Different tilts among the arms of the radial maze did not disrupt the performance of the animals. In fact, when the task was complicated by an interruption, the rats on the maze with variable tilts made even fewer errors than did the ones on the standard radial maze. These results indicate either that tilts in a radial maze were used as additional cues facilitating the discrimination of the eight arms or that the "increased cost" of the visits of the tilted arms resulted in a higher "choice criterion" for tilted arms (see Brown, 1990). However, during the rotation procedure, arm tilting produced no apparent advantage. Thus, the horizontal component of arm direction (related to the extramaze visual cues) appeared to have priority over the tilts, which may have been used simply as intramaze cues, much like objects or patterns (see, e.g., Winocur, 1982). However, it is possible that the use of more dramatically differing slopes, giving access to separate and well-defined levels in the room, might lead to different results. Indeed, in other experiments, rats tested in three-dimensional mazes (cubic maze) accorded a priority to the vertical dimension of space as opposed to the horizontal one (Grobéty \& Schenk, 1992).

The extremely poor performance of the animals on the maze with variable angles was surprising. Not only was the number of errors extremely high in this maze, but the sequence of visitation of the arms appeared to be disorganized. In particular, the number of times the rats changed the transition turn directions in the central arena was almost twice that observed in the other mazes.

The simplest hypothesis that could be advanced to explain the high number of errors in the VA maze would be to attribute these errors to a confusion between the arms separated by small angles (pairs 1-2, 3-4, 5-6), which might be more difficult to discriminate. However, the distribution of the repeatedly neglected arms contradicts this explanation. If two arms are too close together to be discriminated from each other, both will have an equal chance of being neglected in each training session. This would result, during a series of tests, in an equal frequency of neglect for each arm of the pair. But this was not the case. In Experiment 1, all the arms systematically visited last in the VA maze belonged to pairs of arms separated by small angles, but only one arm of each pair was systematically neglected. One neglected arm was on the right side of the pair (Arm 6), whereas the two others were on the left side (Arms 1 and 3). The neglect of these arms could therefore not be attributed to a simple confusion between nearby arms. No bias in the direction of the transition turns could explain this asymmetric distribution either. In other experiments, discriminability between two parallel arms $\left(0^{\circ}\right.$ angle between arms) has been shown to play only a minor role in radial mazes with a pair of parallel arms. For example, enhancing the discrimination between two parallel arms by the addition of different objects (Schenk, Contant, \& Grobéty, 1990), backgrounds (Schenk \& Grobéty, 1992), and tilts (Grobéty, 1990) improved the efficiency of the animals only slightly in these situations.

The rats displayed a relatively strong tendency to chain their visits of the five other arms in the VA maze. Neither intramaze nor extramaze cues differentiated these arms from the other three, since the maze was oriented in the testing room in four different positions, depending on the animal tested. However, each arm's orientation remained unique relative to the internal structure of the maze.

Because the arms selected by the animals as part of this "chain" formed a relatively regular shape (i.e., dividing the $360^{\circ}$ of the space into five angles of approximately the same value), we examined all of the submazes that could be made with a selection of the arms of the VA maze and estimated their regularity. The mean angle separating a given number of arms was always the same, but the standard error of this mean varied with the selection of arms and gave an estimation of the regularity of the shape. The more similar that the angles between the selected arms were, the smaller was the standard error. Figure 4 shows the most regular and irregular shapes possible that were built on a selection of three to eight arms of the VA maze. The most regular shape is formed by five arms (numbers 2, 4, 5, 7, and 8 of Figure 1b). These arms were precisely those most frequently chained by the animals, and the three remaining arms $(1,3$, and 6$)$ were those neglected by the animals. It is therefore likely that the biases observed in the VA maze were caused by a tendency to visit directions as different as possible, subdividing the $360^{\circ}$ of the space as regularly as possible. Such optimization of the distribution of the running directions may have inclined the rats to chain the most distant arms and to neglect those that provided little new information. This hypothesis will be discussed further in the General Discussion.

Experiment 1 shows that a simple horizontal irregularity in the maze increased the complexity of the radial task. In Experiment 2, we decided to test whether a pretraining phase could improve the performance of the animals in the VA maze and eliminate the different biases in patrolling this maze (i.e., neglected arms, number of changes in the transition turn directions, and the tendency to chain the visit of Arms 2, 4, 5, 7, and 8). Therefore, we pretrained two groups of rats on a standard and on a VA maze with a procedure allowing them to visit the maze in two stages, with access to only four arms at a time. This procedure was chosen for two reasons. First, the memory load was lower because the animals faced four arms at a time instead of eight, thus simplifying their 
Shape Examples With Regularity

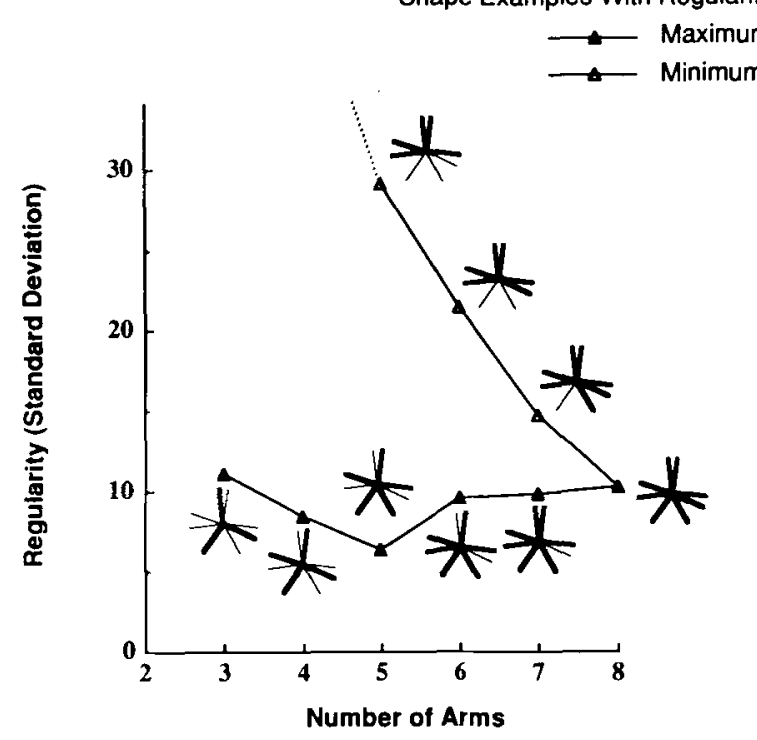

Figure 4. Identification of the most regular shape designed by either $3,4,5,6,7$, or 8 of the arms of the VA maze. To represent the regularity of the mazes, the standard errors of the mean angles delimited by the adjacent arms in each possible selection (shown in bold in the examples) were calculated. The smaller the standard error, the more equal are the angles and, consequently, the more regular is the maze. The two curves represent the standard errors of the most regular and of the most irregular submazes as a function of the number of arms used. No perfectly regular shape (standard errors $=0$ ) could be obtained by using any combination of the eight arms of the VA maze. The five arms numbered $2,4,5,7$, and 8 formed the most regular maze that could be built by selecting among the eight arms of the VA maze.

task. Second, the procedure should have prevented the animals from developing specific arm transitions since all of the arms were not open simultaneously during a trial.

\section{EXPERIMENT 2}

Method
Subjects
The subjects were 12 male hooded Long-Evans rats, 3 to 5 months
old, from our breeding colony. They had been trained in a Morris

place learning task during development (3-5 weeks of age). They were maintained in the same conditions as were the rats used in Experiment 1 .

\section{Apparatus}

The rats were tested on a standard eight-arm radial maze or on a radial maze with variable angles (VA maze) identical to the ones used in Experiment 1 (Figures la and $1 b$ ). Four orientations of the mazes were used, the arm designated as number 1 pointing North, South, East, or West.

\section{Procedure}

"4-4 choice" procedure. The subjects were randomly assigned to two groups, with 6 animals tested on each maze. Prior to each trial, the entrances of four arms were blocked by Plexiglas sliding doors. The blocked arms were chosen in such a way that at least two arms separated by a small angle in the VA maze were open at the same time. This procedure was chosen to encourage visits of two adjacent arms within a small number of choices. The different patterns were chosen randomly for each rat on each trial, and similar patterns were assigned to the rats in the standard maze. The tested animal was placed in the maze and allowed to make choices until every unblocked arm had been visited. It was then removed for $1 \mathrm{~min}$ and reintroduced to complete the maze. In contrast to the procedure used in Experiment 1, the four arms open before the interruption were closed in the second part of the trial, and only the four unvisited arms were open after the interruption. Thus, errors in this phase could include only repeated visits to one of these four arms. This procedure was conducted for 9 consecutive days.

Free-choice procedure. After 9 days of testing with the 4-4 choice procedure, the animals were tested for 15 days with a freechoice procedure as described in Experiment 1.

\section{Results}

Figure 5 shows the performance of the animals in the two types of mazes during the two different training phases. As in Experiment 1, the animals in the VA maze performed poorly compared with those in the standard maze, whatever the procedure [ANOVA 4-4 choice procedure: between-group $F(1,10)=8.43, p=.02$; free-choice procedure: between-group $F(1,10)=7.00, p=.02$ ].

During Trials 10-12 (Block 4) of Experiments 1 and 2 , all of the animals were tested with a free-choice procedure. A comparison of the performance of the two groups tested in the VA maze is therefore possible for these trials. Despite the change in testing procedure at the 10th trial of Experiment 2, which might have disturbed

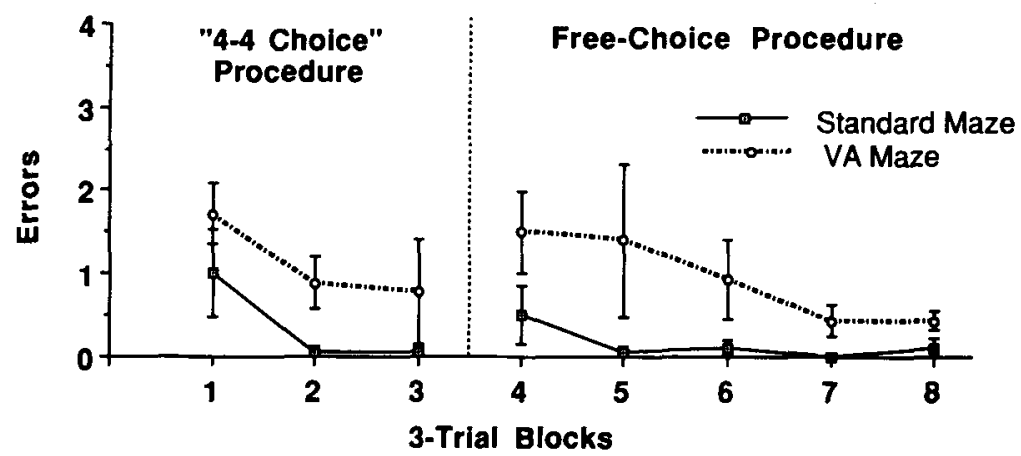

Figure 5. Mean number of errors by blocks of three trials observed on the standard and VA maze during the "4-4 choice" procedure (Block 1-3) and the free-choice phase (Blocks 4-6) of Experiment 2. 
the animals, the mean number of errors was significantly lower in Experiment 2 than in Experiment $1(1.5 \pm 0.49$ vs. $3.29 \pm 0.96$, respectively; Mann-Whitney $Z=1.77$, $p<.05)$. In comparison, the performances of the two groups trained on the standard radial maze were already close to the minimum of errors and therefore did not differ significantly across experiments (mean number of errors for Trials $10-12=0.21 \pm 0.09$ and $0.50 \pm 0.35$ for Experiments 1 and 2, respectively; Mann-Whitney $Z=$ 0.28 , n.s.).

During Trials 10-15, the spatial distribution of the last arm visited in the VA maze did not differ significantly from chance $\left[\chi^{2}(7)=10.76\right.$, n.s.]. The neglected arms of Experiment 1 were now taken last in only $8 \%$ of the sequences for Arm 1, in 17\% for Arm 3, and in 22\% for Arm 6. However, the tendency to chain the visits of the five other arms (calculated as in Experiment 1) appeared in $50 \%$ of the sequences in the VA maze versus $11 \%$ in the standard radial maze $\left[\chi^{2}(1)=12.81, p<.001\right]$. This high frequency was maintained throughout the experiment.

During Trials 10-12, the number of changes between clockwise and counterclockwise turns in the central arena was higher in the VA maze than in the standard maze $(1.55 \pm 0.32$ vs. $0.50 \pm 0.17$, respectively; Mann-Whitney $Z=2.57, p<.01$ ). During the next three trials, however, it decreased rapidly in the VA maze and was no longer significantly higher than in the standard maze $(0.78 \pm 0.22$ vs. $0.44 \pm 0.12$; Mann-Whitney $Z=0.88$, $p=.38$ ).

\section{Discussion}

The pretraining phase helped the rats on the VA maze perform more efficiently in the free-choice trials than did the animals trained only with a free-choice procedure as in Experiment 1. However, the difference in performance between the animals trained in the standard maze and those trained in the VA maze remained significant in both training phases.

During the 4-4 choice procedure, the different selections of four open arms formed an irregular shape in both conditions. The only difference between the two maze conditions was the smaller size of some of the angles separating the arms in the VA maze. Therefore, even when the rats had to face only four arms at a time, the orientation of the arms and the presence of small angles between pairs of arms affected their efficiency.

The use of a 4-4 choice procedure produced an anticipated effect. In the free-choice procedure, a lower level of errors was observed than in Experiment 1, and at least some of the exploratory biases observed in that experiment (such as the arms systematically visited last and the number of changes in the direction of the turns) were less apparent in the free-choice phase of this experiment.

However, despite the pretraining, the tendency on the VA maze to chain Arms 2, 4, 5, 7, and 8 in consecutive choices reappeared during the free-choice procedure of Experiment 2 . Such a spontaneous tendency to chain arms that form a regular shape seems to be very strong, since it appeared even after a pretraining phase during which it could be neither expressed nor developed.

\section{GENERAL DISCUSSION}

The modifications of the shape of the radial maze presented here led to two distinct results. First, the introduction of tilts had no disruptive effect on the performance of the animals, and the different tilts appeared to be used simply as additional intramaze cues. Second, and by contrast, a horizontal distortion of the regular shape of the standard radial maze had a dramatic disruptive effect on the rats' performance. In the VA maze of Experiments 1 and 2, there was a tendency to chain the five arms dividing the space evenly, whereas the three other arms appeared to be neglected. The use of a pretraining procedure that partially constrained the choices reduced the neglect of these three arms but did not decrease the tendency to chain the five most widely separated arms.

The poor results obtained with the animals in the VA maze were clearly related to the presence of small angles between arms. Small angles could be viewed as the source of different problems. First, they could induce confusions between nearby arms. As already discussed in Experiment 1 , the nonrandom choice of the neglected arms showed that a simple confusion between the arms separated by small angles cannot account for the difficulties encountered by the animals. A second problem could have been that the rats avoided sharp turns when going from arm to arm and therefore would not chain two adjacent arms consecutively when the angle between them was too small. However, in Olton et al.'s (1977) 17-arm maze, a regular maze with $21^{\circ}$ angles between arms, rats used adjacent choice strategies with relatively high frequency. Furthermore, under certain conditions in a radial maze with two parallels (a curtain was placed between the two parallel arms), rats were found to visit the two parallel arms consecutively as often as they visited any other pair of arms of the maze (Schenk \& Grobéty, 1992). Thus, there is no absolute motor constraint preventing rats from visiting arms separated by small angles.

In fact, the low score of adjacent choices usually recorded during the first sessions in a radial maze (Grobéty, 1990) or in a modular maze (Schenk et al., 1990) suggests that rats tend to avoid associating visits to adjacent arms in an exploratory phase. This has also been clearly demonstrated in studies of spontaneous alternation behavior in three-arm mazes (see Dember \& Richman, 1989). This behavior could be compared with that in a simpler radial maze, allowing for some procedural differences. In an exploration of the maze, which may or may not be reinforced, rats are stopped after a choice and returned to the start arm for a second choice after a few seconds or minutes of interruption. In such a maze, the choice behavior of the animals depends on the angles between the three arms: The smaller the angle between the two goal arms, the lower the alternation rate (the tendency of the animals to visit the two goals in two consecutive 
choices; Douglas, Mitchell, \& del Valle, 1974; Grobéty, 1990). In addition, it was also found that the smaller the angle between the start and one of the goal arms, the lower the tendency to visit this arm during the first choice and the lower the alternation rate (Schenk \& Grobéty, 1992). Consecutive choices during exploratory behavior are therefore clearly influenced by the relative positions of the arms in a three-arm maze. In fact, even after running in a straight alley, rats placed for a second choice in a $T$ maze tend to run into the goal arm pointing in the direction opposite the one that was enforced by the first run in the straight alley (Sherrick \& Dember, 1966).

All of these results demonstrate a strong tendency for rats to make successive choices in such a way as to run in as many different directions as possible and even to neglect an arm that points in a direction similar to that of the alley they are leaving or have previously visited. The neglect of particular arms in the VA maze may be a consequence of such exploratory behavior. Although the arms separated by small angles may not present a difficuity by themselves for the animals, the contrast between the similarity of direction of these arms and the arms separated by larger angles may incline the animals to run preferentially through the most divergent arms. This bias would explain the rats' tendency to chain the five arms that divided the space evenly and to neglect the arms that pointed in directions similar to the other arms they had already visited.

Such a tendency to spontaneously alternate directions during exploration behavior might be related to the use of the vestibular system and path integration mechanisms in the early stages of exploration, when no mapping of the environment has yet been established. The use of the vestibular system and of kinesthetic information is generally considered to be limited to relatively short paths because of the cumulation of errors (Etienne, 1987; Potegal, 1982). A first exploration of the space in a minimum number of movements, in directions as distinct as possible, might therefore be the most efficient way to collect information on the environment while efficiently keeping track of one's own movements.

Our experiments point out that the performance of the animals on the eight-arm radial maze depends on the relative orientation of the arms. These data extend Brown's (1990) work demonstrating the influence of the length of the arms and the work of Yoerg and Kamil (1982) showing the importance of the size of the central arena. The observed differences in performance due to these three modifications in shape of the radial maze are attributed to the use of different strategies of exploration and cost/ benefit analyses. The length of the arms, the size of the central arena, and the direction of each arm could easily be combined to form different variations of the radial maze. For example, would the neglected arms of an irregular maze be visited more frequently if they were shorter than the other arms? Would a larger central arena reduce the effect of irregularity? These experimental designs would allow us to test the weight of each of these factors and the way in which they interact when combined. Such an approach would therefore help to understand the underlying mechanisms influencing the behavior of normal animals in the radial maze. Whatever the results of such experiments, the use of the radial maze as a simple memory test should already be viewed with cautionneither the spatial dimension of the task nor its connection with exploration and foraging behaviors should be underestimated.

\section{REFERENCES}

Beatty, W. W., \&havalua, D. A. (1980). Spatial memory in rats: Time course of working memory and effect of anesthetics. Behavioral \& Neural Biology, 28, 454-462.

Brown, M. F. (1990). The effects of maze-arm length on performance in the radial-arm maze. Animal Learning \& Behavior, 18, 13-22.

CoOK, R. G., Brown, M. F. (1985). Retroactive interference in rat radial maze performance: The role of point of delay interpolation and the similarity and amount of interpolated material. Animal Learning \& Behavior, 13, 116-120.

Dember, W. N., Richman, C. L. (1989). Spontaneous alternation behavior. New York: Springer-Verlag.

Douglas, R. J., Mitchell, D., \& Del Valle, R. (1974). Angle between choice alleys as a critical factor in spontaneous alternation. Animal Learning \& Behavior, 2, 218-220.

ETIENNE, A. S. (1987). The control of short distance homing in the golden hamster. In P. Ellen \& C. Thinus-Blanc (Eds.), Cognitive processes and spatial orientation in animal and man (pp. 233-251). NATO ASI Series. Dordrecht, The Netherlands: M. Nÿhoff.

Groséty, M.-C. (1990). Importance de la dimension verticale de l'espace locomoteur dans l'orientation spatiale du rat de laboratoire. Unpublished doctoral dissertation, University of Lausanne, Lausanne, Switzerland

GrobEty, M.-C., \& SCHENK, F. (1992). Spatial orientation in 3dimensional mazes. Animal Behaviour, 43, 1011-1020.

Kraemer, P. J., Gilbert, M. E., * InNis, N. K. (1983). The influence of cue type and configuration upon radial-maze performance in the rat. Animal Learning \& Behavior, 11, 373-380.

MAKI, W. S., Brokofsky, S., \& Berg, B. (1979). Spatial memory in rats: Resistance to retroactive interference. Animal Learning \& Behavior, 7, 25-30.

Olton, D. S., Collison, C., Werz, M. A. (1977). Spatial memory and radial arm maze performance of rats. Learning \& Motivation, 8 , 289-314

Olton, D. S., \& SAmuelson, R. J. (1976). Remembrance of places past: Spatial memory in rats. Joumal of Experimental Psychology: Animal Behavior Processes, 2, 97-116.

Potegal, M. (1982). Vestibular and neostriatal contributions to spatial orientation. In M. Potegal (Ed.), Spatial abilities: Development and physiological foundations. London: Academic Press.

RoberTs, W. A. (1979). Spatial memory in the rat on the hierarchical maze. Learning \& Motivation, 10, 117-140.

SChenK, F., Contant, B., Grobétr, M.-C. (1990). Angle and directionality affect rat's organization of visit sequences and spatial learning in modular mazes. Learning \& Motivation, 21, 164-189.

SCHENK, F., GROBETY, M.-C. (1992). Interactions between directional and visual environmental cues in spatial learning by rats. Learning \& Motivation, 23, 80-98.

Sherrick, M. F., Dember, W. N. (1966). Trial-two goal arm 
alternation to direction of movement in trial-one straight alley. Psychonomic Science, 1, 317-318.

WiNOCUR, G. (1982). Radial-arm-maze behavior by rats with dorsal hippocampal lesions: Effects of cueing. Journal of Comparative \& Physiological Psychology, 96, 155-169.

YoerG, S. I., \& KAMIL, A. C. (1982). Response strategies in the radial arm maze: Running around in circles. Animal Learning \& Behavior, 10, 530-534.
Zoladek, L., \& RoberTs, W. A. (1978). The sensory basis of spatial memory in the rat. Animal Learning \& Behavior, 6, 77-81.

(Manuscript received November 12, 1991; revision accepted for publication April 13, 1992.) 